\title{
Certificat optionnel d'anglais médical
}

Compte rendu d'expérience

Ludmilla Delorme

\section{OpenEdition}

\section{Journals}

Édition électronique

URL : http://journals.openedition.org/asp/4370

DOI : $10.4000 /$ asp.4370

ISSN : 2108-6354

Éditeur

Groupe d'étude et de recherche en anglais de spécialité

Édition imprimée

Date de publication : 1 mars 1993

Pagination : 209-220

ISSN : 1246-8185

\section{Référence électronique}

Ludmilla Delorme, « Certificat optionnel d'anglais médical », ASp [En ligne], 1 | 1993, mis en ligne le 29 avril 2014, consulté le 30 avril 2019. URL : http://journals.openedition.org/asp/4370 ; DOI : 10.4000/ asp. 4370

Ce document a été généré automatiquement le 30 avril 2019

Tous droits réservés 


\title{
Certificat optionnel d'anglais médical
}

\author{
Compte rendu d'expérience
}

Ludmilla Delorme

1 Le Certificat optionnel d'anglais médical est une création récente puisqu'il date de la rentrée universitaire 1991. C'est à la demande expresse de certains enseignants de médecine que le Département de langues vivantes pratiques de l'Université de Bordeaux 2, université qui regroupe des enseignements de médecine, pharmacie, biologie, sciences sociales et psychologiques, sport, et (Bordeaux oblige) œnologie, a été amené à organiser cette formation. Un vaste projet d'enseignement de langues avait été élaboré en mai 1990 dans le cadre de la Contractualisation par l'équipe du Département. Il s'agissait de la mise en place d'un ensemble cohérent de formation en langues qui prenait en compte les enseignements déjà existants au niveau de la formation initiale et de certaines maîtrises, mais qui visait également la création d'enseignements optionnels notamment en médecine et en pharmacie et la préparation d'un Diplôme d'Université (DU) mention anglais médical, d'un DESS de communication scientifique en langue anglaise (Dominante $=$ Sciences de la vie et de la Santé) d'un DEA interuniversitaire national, d'anglais de spécialité ainsi qu'un DESS interuniversitaire régional, de formateurs en langues pour la formation continue. Pour l'heure, en ce qui concerne les créations, seuls ont été mis en place le DEA d'anglais de spécialité (novembre 1991) et le certificat optionnel d'anglais médical. Il n'est pas exclu que d'autres formations prennent corps dans un avenir proche étant donné la demande exponentielle formulée par diverses instances universitaires et hospitalières, l'École de santé navale et le Centre hospitalier régional, entre autres.

2 Le Certificat optionnel d'anglais médical s'adresse à des étudiants de DCEM 3 ce qui correspond à la $5^{\mathrm{e}}$ ou $6^{\mathrm{e}}$ année de médecine. Tous titulaires du Certificat de synthèse clinique et thérapeutique (CSCT), ils ont reçu une solide formation théorique. Parallèlement, au cours de la $2^{\mathrm{e}}$ et $3^{\mathrm{e}}$ année de médecine, ils ont passé respectivement trois heures par semaine à l'hôpital pendant huit mois, au cours de la $4^{\mathrm{e}}$ année quatre heures chaque jour pendant huit mois et, enfin, au cours de la $5^{\mathrm{e}}$ et $6^{\mathrm{e}}$ années toutes leurs 
matinées chaque jour sont consacrées à l'hôpital pendant douze mois. Leur expérience pratique grâce aux responsabilités diverses, gardes, urgences, qui leur sont confiées est donc considérable. Ce sont des étudiants sérieux qui se prennent au sérieux.

3 La définition de ce profil d'étudiant appelle deux remarques, l'une en amont concernant la décision institutionnelle de leur offrir de l'anglais à eux, l'autre en aval touchant à leur comportement vis-à-vis du cours.

4 Alors qu'un plan cohérent et continu d'enseignement de langues avait été proposé à partir de la $2^{\mathrm{e}}$ année de médecine, nos collègues médecins ont choisi de privilégier l'étape intermédiaire du cône que représente leur public étudiant. Ces étudiants déjà nantis d'une solide formation théorique et pratique en médecine ont besoin de l'anglais pour lire

On sait en effet l'importance des séances de bibliographie dans tout service hospitalouniversitaire. Ultérieurement, si certains d'entre eux franchissent le cap de l'internat, deviennent chefs de clinique ou praticiens hospitaliers, ceux-là seront amenés à rédiger en anglais et à présenter leurs communications lors de congrès, donc à perfectionner la production écrite et orale. Au niveau du DCEM 3, la finalité immédiate dans le cadre de leurs études se limite à la compréhension de l'écrit.

6 De la part des instances universitaires, la décision de choisir cette tranche d'étudiants apparait assez légitime quoiqu'un peu frileuse par rapport à l'orientation d'ensemble qui se dessine. Il semblerait en effet que le vent de la culture humaniste souffle à nouveau sur les études médicales après les décennies de terrorisme mathématique et technologique puisqu'on voit timidement réapparaître des enseignements de philosophie, de français, voire de théologie au niveau des premiers cycles scientifiques. Dans ce char humaniste aux couleurs d'Arlequin pour reprendre l'image maintenant galvaudée de Michel Serres, l'enseignant de langues, hybride ou métis s'il en est, a plus que jamais sa place et il ne faut pas s'étonner qu'on le sollicite de plus en plus. Le culturel, au moins dans les intentions, reprend vigueur sans que « l'utilitaire » en perde d'importance (Serres 1991).

7 Pour en revenir au cadre et aux contraintes institutionnels, il nous a donc été alloué un bloc de trente heures étalées sur deux mois à raison de deux ou quatre par semaines l'après-midi soit quinze séances de cours à dispenser dans un amphithéâtre.

8 Les étudiants, comme l'intitulé l'indique, ont la faculté de choisir deux certificats optionnels sur les six qui leur sont proposés, à savoir certificat optionnel de : anglais médical, cancérologie pratique, gériatrie, immunologie clinique, radiologie et thérapeutique hydrologique. L'examen qui sanctionne ce certificat est censé durer une heure.

9 Comme on le voit, il s'agit d'une véritable intégration dans un cursus. Le certificat est optionnel certes, mais au même titre que d'autres disciplines dûment reconnues comme telles, et je rappellerai à ce propos la définition que donne Michel Foucault d'une discipline: "Pour qu'il y ait discipline, il faut qu'il y ait possibilité de formuler indéfiniment des propositions nouvelles ».

10 C'est bien en effet l'aspect novateur de cette formation que j'aimerais faire ressortir maintenant en examinant comment l'expérience s'est déroulée à partir des données institutionnelles et humaines qui nous étaient imposées.

11 Début septembre 1991, nous nous sommes retrouvés face à une vingtaine d'étudiants sur les quarante inscrits initialement, l'information ayant été diffusée de façon assez discrète. 
Certains de ces étudiants n'appartenaient pas à l'UFR qui avait inscrit le certificat dans son cursus, mais les responsables des autres UFR se sont ralliés avec empressement à l'initiative, bref un certain flou administratif a caractérisé la mise au point: nous ignorons encore combien d'étudiants passeront l'examen, un quarantaine sûrement, une centaine peut-être, compte tenu des demandes.

Les étudiants dont 75 \% de Français, 25 \% d'étrangers (Libanais en majorité) s'attendaient à un cours d'amphi. Nous les avons placés d'entrée de jeu en situation de découverte de leurs propres besoins en les amenant à se positionner individuellement par rapport à la langue.

excice extrêmement court sous la forme de deux phrases de trois lignes prises au hasard dans une revue médicale a permis aux étudiants de prendre conscience de ce qui pour nous est déjà une antienne (le vocabulaire technique ne fait pas problème pour les étudiants avancés); les difficultés de compréhension viennent de certains aspects du lexique, de la grammaire, de la syntaxe de la langue usuelle, du moins pour ce qui a trait à la compréhension écrite.

14 À ce propos, nous avons constaté immédiatement un premier clivage chez les étudiants selon leurs perspectives d'implantation professionnelle, les étudiants étrangers et certains Français souhaitant travailler surtout l'oral et en particulier les composantes du discours médecin-patient plus que le discours scientifique médical proprement dit.

Par commodité, pour faciliter la coordination entre les trois intervenants et pour satisfaire aux intérêts divers correspondant au degré d'avancement des étudiants dans leurs études médicales, nous avons choisi d'utiliser le manuel de David V. James intitulé Medicine dans la série English for Academic Purposes publié par Cassell en 1989, qui se présente sous la forme de quinze Unités centrées autour d'une discipline ou d'un thème, ce qui correspondait aux quinzeséances que nous devions assurer.

Comme il est écrit sur la $4^{e}$ de couverture, l'accent est mis dans cet ouvrage sur la lecture en vue de recherche d'information et sur la compréhension écrite et également orale puisque deux cassettes accompagnent le manuel, les documents utilisés étant tous prétendument authentiques. Il y est également stipulé à deux reprises que la connaissance du sujet n'est pas un prérequis à son utilisation ni par le professeur ni par les étudiants.

17 Si nous avons été relativement satisfaits du choix de cet ouvrage, nous l'avons cependant sérieusement adapté à notre méthode en allant jusqu'à détourner totalement certains exercices de leur fin initiale, précisément, parce que, contrairement à ce qui est dit plus haut c'est un manuel qui s'adresse à des étudiants déjà très spécialisés dans leur discipline.

18 En guise d'illustration je prendrai pour exemple un exercice d'explicitation de vocabulaire qui se retrouve à chaque chapitre :

Can you explain in simple language the meaning of the following words: standardise; mortality; epidemic; vaccines; manipulation

19 Il est absolument évident qu'aucun de ces termes ne pose problème à des étudiants francophones même s'ils n'ont pas fait de latin ou de grec. En revanche, si on leur demande d'apparier jabs, catching ou handle avec les termes cités ci-dessus, la difficulté surgira et a fortiori si l'on demande une explication "in simple language ». Il faut déjà une bonne maitrise de l'anglais usuel pour dire que mortality is the rate at which deaths take place. La tâche devient redoutable pour l'étudiant français puisque c'est faire le saut de 
l'abstrait familier immédiatement saisi au concret étranger souvent idiomatique dans l'articulation syntaxique. En revanche la prononciation de ces termes d'origine latine ou grecque risquera de présenter quelques difficultés et des exercices appropriés s'imposeront.

Précisément, pour ne pas planer dans le normatif, j'aimerais pour clore ce compte rendu d'expérience, montrer la dynamique non pas propre à chaque cours, car chaque enseignant insuffle sa propre dynamique en fonction de sa personnalité (on n'enseigne jamais que ce que l'on est), mais celle d'un cours particulier. Et, à la faveur de cette esquisse, rappeler quelques principes fondamentaux de l'approche communicative que nous avons en commun appliqués à la mise en œuvre de cette formation.

21 I- Première phase : préfocaliser en demandant aux étudiants de parler pendant une minute, soit en français soit en anglais, de nutrition par exemple : soit en écrivant le mot au tableau soit, en montrant une photo d'obèse ou de dénutrit soit, en faisant écouter un fragment de dialogue du type: Are you hungry ou Do you like chocolate? Il s'agit d'une amorce qui permet un ancrage sémantique en même temps qu'elle indique le bornage du champ conceptuel.

La deuxième phase consiste à faire récapituler par les étudiants les termes ou expressions relatifs au sujet qu'ils ont dû employer pour s'exprimer.

Interview en phase trois : la lecture du texte par l'enseignant, les étudiants prennent des notes. Il s'agit là d'écoute avec repérage d'informations.

Phase 4 : les étudiants comparent leurs notes avec la liste récapitulative et la complètent. Ils sont aussi amenés à opérer une première série d'autocorrections.

Phase 5 : cette activité se solde par un contrôle précis de traduction ponctuelle du français vers l'anglais: ex. régime alimentaire/diet; pénurie/food shortage. Il s'agit là d'une activité lexicale simple, binaire, mais précise, gouvernée par le principe de l' information-gap.

II- Dans un deuxième temps, à l'aide de la $W$ H. grid (who - what - which - when - where how much - how many how long - why) les étudiants à qui un deuxième texte est lu sont invités à reconstruire le sens global du passage et à le reformuler devant leur partenaire qui lui, sera en possession de la moitié du texte, les rôles s'inversant à mi-parcours.

Il y a là reformulation en même temps que vérification simultanée de la production. L'effort n'est plus seulement lexical, mais syntaxique et grammatical. La production est cependant étroitement guidée.

28 III- Troisième temps : Préparation du jeu de rôle, en l'occurrence interview d'un patient obèse par son médecin. L'obèse rédige son propre portrait et tente de faire l'étiologie de son obésité. Le médecin prépare ses questions.

29 Un intermède grammatical peut donner lieu à la révision rapide de la forme interrogative de l'emploi du présent simple et du présent continu.

L'écoute d'un extrait de dialogue de type interview permet de travailler sur l'intonation en mode interrogatif et sur les groupes de souffle.

31 L'activité se clôt par la réalisation du jeu de rôle lui-même qui intervient à la fois comme contrôle de toutes les connaissances acquises pendant la séance et comme détente ludique. 

lettre du médecin spécialiste à son correspondant, ce qui impliquera de la part du rédacteur la transposition en langage scientifique des notions exprimées oralement en langage vulgaire. La boucle est ainsi refermée et l'exercice théoriquement gratifiant (Grellet 1991) puisqu'il permet de se retrouver dans la terra cognita du discours scientifique. Idéalement nos étudiants produiront du Georges Perec, ce petit livre délectable d'une époustouflante drôlerie que tout spécialiste d'ESP rédaction scientifique se doit d'avoir lu (Perec 1991).

Pour poursuivre et conclure dans la note optimiste, j'évoquerai le satisfecit d'ensemble qui nous a été décerné par les étudiants lors de la rédaction des bilans et qui tient en deux mots clés : sentiment de découverte et plaisir. Ces mots clés sont bien entendu liés au principe fondateur de l'approche communicative qui centre le cours sur l'apprenant et ses ressources dormantes que l'enseignant s'efforce d'activer par la multiplicité des démarches heuristiques, dans une volonté constante d'adaptation à l'auditoire si hétérogène soit-il dans ses souhaits, en maintenant dans la dynamique du cours un tempo joyeux et soutenu et une variété suffisante pour ne laisser jamais s'installer l'inacceptable ennui.

Il me paraît très significatif que, dans nombre d'institutions à vocation scientifique en particulier, au niveau des DEUG, de biologie par exemple, dans le concours d'entrée de certaines universités de médecine (tours) et éventuellement au niveau de certains certificats DCEM 1 ou 2, voire de DU, les enseignants, non littéraires fassent introduire des modules dits d'expression voire de compréhension qui n'ont, eu soi, rien à voir avec la discipline enseignée (médecine, biologie, psychologie, sociologie, etc.)

Il s'agit manifestement là d'un constat de déficience; rompus, plus ou moins, aux mécanismes décharnés du raisonnement scientifique ou pseudo scientifique, la majorité de ces étudiants ne savent plus s'exprimer, peut-être même ne savent-ils plus comprendre le texte. Ils saisissent le sens des graphiques, des courbes, des schémas, des images - je pense en particulier à tout le poids de l'imagerie médicale - ils ne savent plus interpréter ni pour eux-mêmes ni pour autrui, le texte n'est plus qu'enluminure.

Que leur manque-t-il et en quoi diffèrent-ils de la génération de leurs parents ou de leurs grands-parents pour que ces derniers éprouvent impérativement la nécessité de remédier formellement à cette lacune ? On pourrait penser qu'il s'agit d'une espèce de nostalgie de la rhétorique d'antan, mais force nous est fait, au contact de la dure expérience quotidienne de constater les faits.

Le matraquage médiatique et en particulier télévisuel est sans doute une des causes de ces lacunes. Non seulement beaucoup d'enfants ne lisent plus, mais on ne leur lit plus d'histoires. Le conteur, mobilisateur d'attention a disparu des chaumières et les capacités de raisonnement linéaire, séquentiel, de la tête des enfants en même temps que le pouvoir d'attention. Aujourd'hui, on zappe. Les grands-parents, liseurs ou conteurs ne font plus partie des foyers urbains mono nucléaires, le poste de radio, surtout dans l'univers enfantin a cédé le pas à la télévision ou au magnétoscope voire le magnétophone.

Sans doute les enfants communiquent dans leur langue comme dans la langue étrangère, par onomatopées, par holophrases (par tournure idiomatiques) ; Elen Bialystok a fort bien rendu compte, au cours du séminaires de linguistique que j'ai évoqués dans l'introduction, des trois modes d'approches d'une langue étrangère, le biologique, le 
linguistique et le cognitif. Elle a montré qu'au cours de l'apprentissage d'une langue seconde, l'enfant se montrait supérieur à l'adulte sur le plan phonologique et sur ce plan là uniquement avec néanmoins des variations étonnantes quant à l'âge critique optimum de reconnaissance des phonèmes. D'après les études faites par les psychologues le bébé de quelques jours opère des distinctions plus subtiles parmi les phonèmes que l'enfant de deux ans et il apparaît aussi que la puberté joue un rôle très préjudiciable au décodage phonétique et on en conclut que l'âge optimum de l'apprentissage linguistique se situe entre 10 et 15 ans (Scovel 1988).

Exposés intensément au bruit et à la fureur des impressions visuelles et sonores nos enfants, nos étudiants sont déroutés par l'agencement des mots écrits.

Le débat initié par Chomski de l'existence d'une grammaire universelle n'est certes pas clos et l'on ne saura peut-être pas avant longtemps si les schémas sont de l'ordre de l'inné et de l'acquis, mais il est sûr que les pratiques pédagogiques un peu sauvages qui nous ont amenés (nous les enseignants) à plongés nos petits dans un bain linguistico-littéraire sans préparation, a causé bien des déboires. À ne plus vouloir leur donner des bouées de métalangues, nous ne sommes pas sûrs de leur avoir appris autre chose qu'à barboter dans la fange des morphèmes. Foin des langues mortes, vive les mathématiques! Au pilori l'analyse grammaticale et l'analyse logique d'autrefois, moyennant quoi nos étudiants ne savent plus ce qu'est qu'un nom, un adverbe ou une préposition.

41 Allez donc, privé de ces outils élémentaires d'explication, leur faire comprendre ce qu'est la -ing form en anglais clairement appelée nom verbal ou gérondif du temps du latin de papa! À nous, universitaires, de réparer les dégâts en l'occurrence de retourner à la syntaxe pour faire comprendre les articulations d'un texte - Dieu merci aujourd'hui le fluo marqueur existe.

L'approche cognitive est la $3^{\mathrm{e}}$ voie et les adultes, étudiants et professionnels confondus prennent là décidément le pas sur les enfants. Ils en savent plus et cela les aide. Motivés, ils accrochent du sens aux mots. Intéressés, ils lisent - et je dirais volontiers cela comme Roméo découvrant que Juliette est douée de parole "She speak». Nos étudiants lisent quand nous savons leur fournir des lectures intéressantes et nous passer parfois des manuels scolaires.

Il faut pour cela être à l'affût des thèmes mobilisants et savoir renoncer à évoquer l'esthétique chez Kant le jour où éclate la guerre du Golfe ou sortir un extrait des Enfants de minuit. Quand Khomeiny condamné à mort l'auteur des Versets sataniques, en d'autres termes savoir enseigner compréhension et expression en demeurant au plus près et du monde et de l'auditoire - l'enseignant n'est-il pas d'abord et seulement le facilitateur de l'apprentissage, le reste est une autre histoire.

Scovel, Thomas. 1988. A Time to Speak: A psycholinguistic inquiry into the critical period for human speech. New York : House/Harper \& Row. 


\section{BIBLIOGRAPHIE}

Grellet, Françoise. 1991. Apprendre à Traduire. Nancy : Presses Universitaires de Nancy.

Perec, Georges. 1991. Cantatrix Sopranica L. et autres écrits scientifiques. Paris : Seuil.

Serres, Michel. 1991. Le Tiers-Instruit. Paris : Ed-F-Bourin.

\section{AUTEUR}

\section{LUDMILLA DELORME}

Ludmilla Delorme est maître de conférences au DLVP, Université de Bordeaux 2.

ludmilla.delorme@free.fr 